Ciencia y Educación, Vol. 5, No. 3, septiembre-diciembre, 2021

ISSN (impreso): 2613-8794・ISSN (en línea): 2613-8808

DOI: https://doi.org/10.22206/cyed.2021.v5i3.pp117-127

\title{
Contribuciones de la Neuropsicología a nivel educativo: un análisis teórico y reflexivo
}

\author{
Contributions of Neuropsychology at an Educational \\ Level: A Theoretical and Reflexive Analysis
}

Jorge Camilo Rhenals-Ramos a ORCID: 0000-0001-8851-2737

Recibido: 24/04/21 Aprobado: 28/06/21

Cómo citar: Rhenals-Ramos, J. C. (2021). Contribuciones de la Neuropsicología a nivel educativo: un análisis teórico y reflexivo. Ciencia y Educación, 5(3), 117-127. Doi: https://doi.org/10.22206/cyed.2021.v5i3.pp117-127

\section{Resumen}

La Neuropsicología presenta elementos importantes a favor del aprendizaje. Su conocimiento ofrece posibilidades para afrontar el trabajo formativo con estudiantes desde una perspectiva de trabajo diferenciada; sin embargo, a nivel escolar aún se desconocen algunas de sus contribuciones más importantes. Este estudio describe teóricamente algunos aportes de la Neuropsicología al aprendizaje, reflexionando sobre su implementación a nivel educativo. El abordaje de esta disciplina ha facilitado el conocimiento neurocientífico del aprendizaje, potenciando las características individuales desde procesos de ajuste educativo y fortaleciendo los procesos inclusivos en el aula. La comprensión de diversas alteraciones relacionadas con el aprendizaje desde la perspectiva neuropsicológica aporta información teórica y elementos empíricos que buscan ajustar metodologías, procesos y programas para el mejoramiento educativo. Sus contribuciones reflejan una oportunidad importante de avance a partir del conocimiento de las dificultades neuropsicológicas de los alumnos y las mejores formas de actuar a nivel educativo.

Palabras clave: Neuropsicología, educación, aprendizaje, aportes educativos, éxito académico.

\begin{abstract}
Neuropsychology shows important elements in favor of learning processes; its knowledge enables the possibility of facing formative work with students from a differentiated work standpoint. At an educational level, however, some of its most important contributions are still unknown. This research theoretically describes some contributions of Neuropsychology to the process, making a reflection on its implementation at an educational level. The approach to this discipline has facilitated the neuroscientific knowledge of learning, boosting the individual characteristics from educational adjustment processes and strengthening the inclusion processes in the classroom. The understanding of several alterations relating to students' learning from a neuropsychological perspective provides theoretical information and empirical elements intended to adjust methodologies, processes, and programs for improving education. Its contributions are an important opportunity to advance from the knowledge of students' neuropsychological difficulties and better ways of acting at an educational level.
\end{abstract}

Keywords: Neuropsychology; learning; education; educational contributions; academic success.

a Universidad de Córdoba, Colombia. Correo-e: jorgerhenalsr@correo.unicordoba.edu.co 


\section{Introducción}

Los cambios ocurridos en los últimos años muestran necesidades evidentes relacionadas con las transformaciones educativas, especialmente en la forma como se enseña y se aprende. Estas apuestas curriculares develan la necesidad de afrontar los procesos educativos desde el estudio de la dinámica cerebral como factor transcendental para el éxito educativo.

Los avances propuestos por la Neurociencia han evidenciado nuevos métodos de trabajo asociados a la prevención y al desarrollo académico de los estudiantes (Araya-Pizarro \& Espinoza-Pastén, 2020; Lozoya-Meza et al., 2018). En estos procedimientos se toma como punto de partida las características de los alumnos, analizando limitaciones y desarrollando sus potencialidades como base importante para el aprendizaje, favoreciendo así los procesos de inclusión educativa.

Desde la perspectiva educativa, la Neuropsicología como disciplina neurocientífica presenta elementos importantes, como apuesta para el desarrollo de los aprendizajes. Para Martín-Lobo (2015), los aportes neuropsicológicos a nivel escolar están principalmente relacionados con la prevención de dificultades, la mejora del potencial educativo y la atención a las necesidades formativas de los alumnos. Todos estos aspectos muestran contribuciones importantes de esta disciplina a favor de los procesos educativos.

Sin embargo, en gran medida en los entornos escolares aún se desconocen algunos de los aportes que la Neuropsicología ofrece, demostrando una brecha importante entre los procesos escolares y las afectaciones neuropsicológicas de los estudiantes (Ashton, 2015). Todo esto limita en algún grado el conocimiento del docente sobre las características de los estudiantes, así como las mejores formas de actuar a nivel educativo.

En este sentido, se considera importante ahondar sobre las implicaciones educativas que presenta la Neuropsicología a nivel escolar, como disciplina que aporta al desarrollo de los procesos educativos en los estudiantes. Este estudio tiene como objetivo principal abordar teóricamente algunos aportes de la Neuropsicología al aprendizaje, reflexionando sobre su implementación a nivel educativo.

\section{Aportes de la Neuropsicología a nivel educativo}

La Neuropsicología como disciplina presenta su génesis a nivel de médico, clínico y neurológico. En los últimos años se han destacado en la literatura científica elementos importantes a nivel escolar, relacionando la dinámica cerebral y el aprendizaje desde diversos ámbitos de intervención (Ibáñez-Azorín et al., 2018; Martín-Lobo, 2015).

Desde su perspectiva de estudio, esta disciplina analiza el desarrollo de las funciones corticales o cognitivas superiores (FCS) en los seres humanos (RufoCampos, 2006), entendiendo que su procesamiento nos diferencia de otras especies a nivel cognitivo. El objeto de la Neuropsicología es estudiar asociaciones entre las FCS y el Sistema Nervioso (SN) (Rufo-Campos, 2006). Desde el ámbito educativo, queda claro que su desarrollo es indispensable para aportar a partir de fundamentos teóricos o prácticos a los procesos relacionados con el aprendizaje escolar.

La Neuropsicología aporta favorablemente al desarrollo educativo de los alumnos desde el ajuste y la mediación de dificultades asociadas al procesamiento de las FCS (gnocias, praxias, lenguaje y funciones ejecutivas) y su relación con las actividades escolares. El desarrollo de las FCS es un proceso individual y complejo que necesita de la consolidación de etapas madurativas que dependen de factores genéticos, interacciones o experiencias con el medioambiente. Las FCS permiten, a través de la repetición, el desarrollo de tareas complejas desde la ejecución de habilidades más simples (Glejzer et al., 2017). El perfeccionamiento de estas tareas se corresponde con mayores niveles de destreza en el desempeño de actividades escolares como la lectura y la escritura.

$\mathrm{Al}$ respecto, Valencia-Echeverry et al. (2020) establecen que gran parte de las dificultades escolares 
están relacionadas con limitaciones en el desarrollo de habilidades gnósico-práxicas. Las Gnosias son habilidades que se relacionan con la capacidad de percibir e interpretar información sensorial del contexto (Valencia-Echeverry et al., 2020). Su desarrollo respalda la ejecución de diversas tareas esenciales para el éxito académico. Por su parte, las Praxias se asocian con la producción de movimientos de ejecución voluntaria, orientados al desarrollo de tareas motoras a nivel cortical. Estudios recientes sobre dificultades en la ejecución motora también sugieren limitaciones en el rendimiento académico de estudiantes (Higashionna et al., 2017; Macdonald et al., 2018). La disfunción en el desarrollo de estas habilidades puede acarrear dificultades relacionadas con el aprendizaje a nivel escolar.

\section{Intervención neuropsicológica a nivel educativo}

La Neuropsicología ofrece un método concreto de trabajo para adquirir información relacionada con el SN y así comprender profundamente cómo se desarrollan los procesos cognitivos y de aprendizaje. Los aportes científicos que esta disciplina ofrece representan una contribución importante para el avance educativo (Murphy \& Benton, 2010).

Desde la perspectiva de diversas investigaciones, a nivel general se pueden establecer categorías relacionadas con las áreas de intervención neuropsicológica a nivel educativo. $\mathrm{Al}$ respecto, los estudios de Blanco-López et al. (2017) y Martín-Lobo (2015) establecen áreas de trabajo especialmente en el funcionamiento ejecutivo, los procesos de lectoescritura y psicomotores, la lateralización cerebral, el desarrollo perceptivo y las habilidades de pensamiento. El desarrollo de estas habilidades presenta elementos importantes y diferenciadores a favor del rendimiento de los alumnos.

\section{Funcionamiento ejecutivo y aprendizaje}

El funcionamiento ejecutivo es considerado como un proceso esencial para el progreso académico de los estudiantes; el desarrollo de estas funciones se corresponde con un nivel elevado de madurez de las FCS (Fonseca-Estupiñan et al., 2016). Al respecto, los estudios recientes sobre neuroimagen sugieren ampliamente las implicaciones del córtex prefrontal en el desarrollo de estas funciones cognitivas de orden superior (Fuster, 2017; Keenan et al., 2019).

En investigaciones preliminares se sugiere que las dificultades en el desarrollo de las funciones ejecutivas pueden estar acompañadas de patologías del SN, así como de otros trastornos cognitivos (Janke et al., 2014; Ventura et al., 2018), presentando repercusiones negativas que afectan la calidad de vida, la personalidad y el rendimiento escolar.

El desarrollo de las funciones ejecutivas “...permite que un individuo aprenda, recuerde nueva información, integre y actúe sobre información nueva rápidamente, cambie fácilmente entre diferentes actividades, planifique y organice tareas" (Ventura et al., 2018, p. 120). Los procesos ejecutivos comprenden el desarrollo de habilidades cognoscitivamente complejas (organización, planificación, autorregulación, memoria y resolución de problemas), consideradas necesarias para el avance a nivel educativo (Vasquez \& Marino, 2021). El desarrollo de estas habilidades es fundamental para el avance académico de los alumnos. El apoyo del profesorado se considera esencial para su fortalecimiento en el aula (Keenan et al., 2019).

En estudios preliminares, Di Lieto et al. (2017), Frank et al. (2021) y Vasquez \& Marino (2021) respaldan la importancia de intervenciones educativas a nivel ejecutivo como aporte para el avance en los procesos académicos. El desarrollo de estas funciones cognitivas es considerado un proceso esencial para la vida académica de los alumnos, debido a su relación con el rendimiento y el éxito académico (Ahmed et al., 2019; Magalhães et al., 2020; Ventura et al., 2018).

\section{Procesos de desarrollo de la lectoescritura}

El desarrollo de los procesos que involucran actividades de lectoescritura ha sido ampliamente abordado 
desde la Neuropsicología. Su estudio ha facilitado la comprensión a nivel educativo a partir del análisis de diversas alteraciones relacionadas con la adquisición y el procesamiento del lenguaje. Esto ha permitido el desarrollo de distintos métodos para favorecer el aprendizaje de la lectoescritura como proceso vital para el rendimiento de los estudiantes (Duarte-Torrado et al., 2018), así como la comprensión de relaciones entre aspectos neuromotores, visuales, auditivos y medioambientales en la ejecución de estas habilidades (MartínLobo, 2015).

Desde la literatura, se reportan diferentes estudios que analizan alteraciones relacionadas con el deterioro de la función lectoescritora. Estos hallazgos permiten aportar información relevante sobre las características que presentan los estudiantes con estas dificultades y así poder intervenir adecuadamente.

Los estudios de Becker et al. (2017) y Cruz-Rodrigues et al. (2014) reportan que el desarrollo de estas habilidades puede estar afectado por factores genéticos o medioambientales; del mismo modo, las investigaciones de Kuhl et al. (2020) reportan que estas dificultades pueden tener su origen en la maduración atípica de redes neuronales asociadas al lenguaje.

A partir de todo lo expuesto, es necesario considerar la intervención en el entorno educativo como actividad vital para el desarrollo y el éxito académico de los estudiantes; todo esto debido a que desde el ámbito de la intervención neuropsicológica se puede dar respuesta oportuna a estas dificultades educativas, tal como ha sido reportado en diversas investigaciones (Galuschka et al., 2020; Ibáñez-Azorín et al., 2018; Roberts et al., 2020).

\section{Desarrollo psicomotor y lateralización cerebral}

Los procesos psicomotores y de lateralización cerebral forman parte importante del desarrollo integral de cada individuo. Estos procesos aportan a la maduración del SN generando automatismos para el desarrollo de actividades escolares como el dibujo, la lectoescritura y las matemáticas (Barrero-Borrallo et al., 2015; Blanco-López et al., 2017; Martín-Lobo, 2015). La ejecución de estas habilidades también puede ser un predictor importante para identificar posibles dificultades relacionadas con la inmadurez del SN (De Bock et al., 2017; Di Rosa et al., 2016; Senese et al., 2020).

Desde el abordaje neuropsicológico, los procesos psicomotores han sido ampliamente estudiados, dejando clara su vinculación directa con diversas habilidades relacionadas con el aprendizaje escolar (Belén Sáez-Sánchez et al., 2021; Martín-Lobo, 2015). Todas estas consideraciones muestran un panorama importante para intervenir a favor del aprendizaje de los alumnos.

La literatura reciente muestra evidencias empíricas, así como reportes teóricos sobre el desarrollo de diversas intervenciones psicomotoras a nivel educativo. Los hallazgos de Da Silva et al. (2017), Fernandes et al. (2019) y Santner et al. (2018) muestran mejoras importantes en habilidades de tipo motor y cognitivo; del mismo modo, los estudios de Donnelly et al. (2016), ElGarhy \& Liu (2016) y Watson et al. (2017) también aportan evidencia y resultados favorables en el desarrollo corporal, las tareas cognitivas y el rendimiento académico, posterior al desarrollo de intervenciones psicomotoras. Todos estos hechos reflejan una oportunidad importante de avance y ajuste de diferentes dificultades educativas.

\section{Desarrollo perceptivo y aprendizaje escolar}

La percepción es un proceso natural que involucra la capacidad de recibir e interpretar información del entorno. Su importancia para el desarrollo de diversas habilidades a nivel educativo es innegable. En este proceso se involucran los sentidos como mecanismo para captar, extraer y seleccionar información externa que luego será procesada por la actividad del SN; al respecto, Merchán-Price \& Henao-Calderon (2011) dejan claro que la percepción es un proceso mediado por receptores y circuitos nerviosos interconectados que establecen relaciones a partir de los distintos sistemas sensoriales (visual, auditivo, táctil).

Para Balado et al. (2017), el aprendizaje de la lectoescritura requiere de la presencia de diferentes destrezas relacionadas con las habilidades perceptivas auditivas y visuales. Del mismo modo, Ison \& Korzeniowski (2016), con relación a los procesos preceptivos 
visuales, establecen que estos juegan un papel importante para el desarrollo de actividades relacionadas con el aprendizaje escolar. La percepción visual involucra el desarrollo de tareas complejas a partir de un espacio determinado. Estas habilidades son comúnmente utilizadas en diversas actividades a nivel educativo (lectura, escritura, dibujo).

En cuanto a la percepción auditiva, esta función es considerada indispensable para algunas tareas cognitivas y de desarrollo escolar. Moore et al. (2010) estableció relaciones entre este tipo de percepción y la memoria de trabajo en escolares. Estas habilidades asociadas al desarrollo de la percepción auditiva presentan una relación importante con el aprendizaje de actividades necesarias a nivel educativo. Al respecto, Martín-Lobo (2015) aporta claridad sobre las dificultades referidas a la percepción auditiva de sonidos y palabras. Estos aspectos limitan el normal desarrollo de las actividades de lectoescritura, afectando el aprendizaje y el rendimiento escolar.

Los procesos perceptivos dependen de los estímulos y las sensaciones que se reciben del contexto, en donde la estimulación sensitiva juega un papel de crucial importancia. En este proceso se utilizan las vías sensoriales para enviar información a diferentes áreas corticales implicadas en el desarrollo de habilidades utilizadas a nivel escolar (Sotomayor-León et al., 2020).

A través del desarrollo de diversas tareas de percepción sensorial, visual y auditiva se presentan distintas activaciones a nivel cortical (Jassim et al., 2021). Estos procesos se relacionan con la adquisición de distintas habilidades consideradas esenciales para el desarrollo educativo, encontrando bien establecida la labor de la corteza prefrontal en los procesos perceptivos de orden superior y desarrollo cognitivo (Jassim et al., 2021).

En estudios recientes se sugieren relaciones entre estas habilidades de desarrollo perceptivo y los procesos de lenguaje (Ganapathy-Sankar \& Monisha, 2020; Werker, 2018), además en habilidades motoras y sociales (Libertus et al., 2016; Libertus \& Hauf, 2017), el desarrollo atencional y el rendimiento escolar (Clark et al., 2021; de Waal et al., 2018). Todas estas consideraciones develan la importancia del desarrollo perceptivo a nivel educativo.

\section{Habilidades de pensamiento y aprendizaje}

El estudio de las causas recurrentes de dificultades educativas y bajo rendimiento escolar se ha asociado en los últimos años a diversos factores neuropsicológicos. Este tema ha sido abordado constantemente en diversas investigaciones, detallando gran complejidad y variedad de causas que inciden principalmente en el desarrollo académico, social y psicológico de los alumnos (Padua-Rodríguez, 2019).

A partir de este análisis, se han registrado en distintas investigaciones reportes sobre carencias importantes en el desarrollo de habilidades de pensamiento, demostrando la influencia que presentan en el desempeño escolar (Martín-Lobo, 2015). El desarrollo de modelos y programas educativos a partir de la Neuropsicología supone un avance importante para el desarrollo educativo de estas habilidades.

"Las habilidades cognitivas se refieren a las distintas habilidades intelectuales demostradas por los individuos al desarrollar una tarea; esto le permite al sujeto apropiarse del conocimiento para resolver problemas y transformar su entorno" (Araya-Ramírez, 2014, p. 2). Su ejecución a nivel educativo es esencial en distintas actividades académicas necesarias para el avance educativo.

Aunque los reportes de alteraciones en estas habilidades a nivel escolar se han incrementado considerablemente en los últimos años (Araya-Ramírez, 2014; Hedenius et al., 2020; Siddiqua et al., 2020), los avances neurocognitivos han demostrado que los circuitos nerviosos pueden readaptarse a partir de diversas intervenciones, logrando mejoras significativas en su desarrollo (Elaklouk \& Zin, 2018).

Distintos autores han evidenciado que las capacidades cognitivas son el resultado del funcionamiento y la estimulación de diversas habilidades de orden complejo (Glejzer et al., 2017; Vasquez \& Marino, 2021). Los programas de intervención neuropsicológica son alternativas importantes para el desarrollo y la estimulación de habilidades cognitivas de uso frecuente en las actividades escolares.

Desde los aportes de la Neuropsicología, a nivel educativo se han reportado hallazgos importantes que 
dan luces para comprender la mejor forma de actuar ante las limitaciones relacionadas con el aprendizaje y de esta forma redireccionar procesos, programas e intervenciones para el avance educativo y el éxito académico de los estudiantes.

Todas estas aportaciones invitan también a considerar la integración de las disciplinas neurocientíficas al currículo de las instituciones de Educación Superior en la formación inicial del profesorado como alternativa de avance educativo desde los procesos formativos (Calzadilla-Pérez, 2017).

\section{Conclusiones}

Los aportes de la Neuropsicología a nivel educativo han sido tema de creciente interés en los últimos años. Las dificultades ocurridas en el contexto escolar exponen la necesidad de ahondar en sus aplicaciones en el aula, como factor de cambio, transformación y ajuste educativo, relacionando la dinámica cerebral con los procesos de aprendizaje y aportando elementos a favor del éxito académico de los estudiantes.

Las contribuciones de esta disciplina al aprendizaje se asocian a la prevención de diversas dificultades, mejorar el potencial educativo y atender las necesidades formativas de los alumnos. Estos aportes ofrecen la posibilidad de fortalecer los procesos inclusivos en el aula, garantizando derechos e igualdad de oportunidades para todos los estudiantes.

Todos estos aportes también ofrecen la posibilidad de afrontar el trabajo formativo con los estudiantes desde una perspectiva de trabajo diferenciada, a partir del conocimiento neurocientífico del aprendizaje, potenciando las características individuales desde procesos de ajuste educativo. Asimismo, se ven especialmente relacionados con intervenciones educativas en el funcionamiento ejecutivo, procesos de lectoescritura, desarrollo psicomotor, lateralización cerebral, procesos perceptivos y habilidades de pensamiento.

El conocimiento sobre las aportaciones de la Neuropsicología a nivel educativo ofrece la posibilidad de afrontar el trabajo formativo con los estudiantes desde una perspectiva de trabajo diferenciada, a partir del conocimiento científico de los procesos nerviosos y el aprendizaje de los alumnos. Su estudio ha facilitado la comprensión y el análisis de diversas alteraciones desde aportaciones teóricas y prácticas relacionadas con el aprendizaje de los estudiantes y el ajuste de dificultades educativas.

El abordaje de esta disciplina ha permitido el desarrollo de diferentes metodologías teniendo en cuenta las particularidades, las limitaciones y las fortalezas de los estudiantes como procesos vitales para el desarrollo educativo, el avance en los procesos inclusivos y el rendimiento en la escuela.

A nivel curricular, en la formación del profesorado en las instituciones de educación superior se considera importante tener en cuenta estas contribuciones, debido a que ofrecen información relacionada con la comprensión, la identificación temprana y la intervención oportuna en dificultades relacionadas con el aprendizaje escolar.

Todas las consideraciones anteriores reflejan una oportunidad de avance y ajuste de diferentes dificultades educativas, además de que aportan evidencia importante con relación al aprendizaje de los alumnos y dejan claridad sobre el papel de la Neuropsicología a favor del desarrollo de los procesos educativos.

\section{Referencias}

Ahmed, S. F., Tang, S., Waters, N. E., \& Davis-Kean, P. (2019). Executive function and academic achievement: Longitudinal relations from early childhood to adolescence. Journal of Educational Psychology, 111(3), 446-458. https://doi. org/10.1037/edu0000296

Araya-Pizarro, S. C. \& Espinoza-Pastén, L. (2020). Aportes desde las neurociencias para la comprensión de los procesos de aprendizaje en los contextos educativos. Propósitos y Representaciones, 8(1), 312. https://doi.org/10.20511/pyr 2020.v8n1.312

Araya-Ramírez, N. (2014). Las habilidades del pensamiento y el aprendizaje significativo en matemática de escolares de quinto grado en Costa Rica. Actualidades Investigativas En Educación, 14(2). https://doi.org/10.15517/aie.v14i2.14744 
Ashton, R. (2015). Educational Neuropsychology. In Neuropsychological Rehabilitation of Childhood Brain Injury (pp. 237-253). Palgrave Macmillan UK. https://doi.org/10.1057/9781137388 223_12

Balado, C., Maria Rivas, R., Torres López, S., \& María Taboada, E. (2017). Neuropsychological evaluation of learning disabilities in the classroom. 01, 2386-7418. https://doi.org/10.17979/reipe. 2017.0.01.2485

Barrero-Borrallo, M., Vergara Moragues, E. \& MartínLobo, M. P. (2015). Educación Matemática en la Infancia Indicadores competenciales: un instrumento para la mejora del desarrollo de la competencia matemática. Edma 0-6. Educación Matemática En La Infancia, 4(2), 22-31. http:// www.edma0-6.es/index.php/edma0-6

Becker, N., Vasconcelos, M., Oliveira, V., Santos, F. C. Dos, Bizarro, L., Almeida, R. M. M. D., Salles, J. F. De, \& Carvalho, M. R. S. (2017). Genetic and environmental risk factors for developmental dyslexia in children: Systematic review of the last decade. In Developmental Neuropsychology (Vol. 42, Issues 7-8, pp. 423-445). Routledge. https://doi.org/10.1080/8756564. 2017.1374960

Belén Sáez-Sánchez, M., Gil-Madrona, P. \& Martínez-López, M. (2021). Desarrollo psicomotor y su vinculación con la motivación hacia el aprendizaje y el rendimiento académico en Educación Infantil. 392, 177-203. https://doi.org/10.4438/1988592X-RE-2021-392-483

Blanco-López, J. L., Miguel-Pérez, V., García-Castellón Valentín-Gamazo, C. \& Martín-Lobo, P. (2017). Neurociencia y Neuropsicología educativa - Publicaciones - Ministerio de Educación y Formación Profesional. Secretaría General Técnica. Centro de Publicaciones. Ministerio de Educación, Cultura y Deporte. https://sede.educacion.gob.es/publiventa/neurociencia-y-neuropsicologia-educativa/ educacion-psicologia/22103

Calzadilla-Pérez, O. O. (2017). La integración de las neurociencias en la formación inicial de docentes para las carreras de la educación inicial y básica: caso Cuba. Actualidades Investigativas En Educación, 17(2). https://doi.org/10.15517/ aie.v17i2.28709

Clark, E., Tullo, D., \& Bertone, A. (2021). Perceptual reasoning skills mediate the relationship between attention and math proficiency in individuals with a neurodevelopmental condition. Research in Developmental Disabilities, 111. https://doi. org/10.1016/j.ridd.2021.103880

Cruz-Rodrigues, C., Barbosa, T., Toledo-Piza, C. M. J., Miranda, M. C., \& Bueno, O. F. A. (2014). Neuropsychological characteristics of dyslexic children. Psicologia: Reflexao e Critica, 27(3), 539-546. https://doi.org/10.1590/16787153.201427315

Da Silva, A. Z., Pereira, F. L. H., Mincewicz, G., de Araujo, L. B., Guimarães, A. T. B., \& Israel, V. L. (2017). Intervenção Psicomotora para estímulo do Desenvolvimento Motor de escolares de 8 a 10 anos. Revista Brasileira de Cineantropometria e Desempenho Humano, 19(2), 150-163. https:// doi.org/10.5007/1980-0037.2017v19n2p150

De Bock, F., Will, H., Behrenbeck, U., Jarczok, M. N., Hadders-Algra, M., \& Philippi, H. (2017). Predictive value of General Movement Assessment for preterm infants' development at 2 years - implementation in clinical routine in a non-academic setting. Research in Developmental Disabilities, 62, 69-80. https://doi.org/ 10.1016/j.ridd.2017.01.012

De Waal, E., Pienaar, A. E., \& Coetzee, D. (2018). Influence of Different Visual Perceptual Constructs on Academic Achievement Among Learners in the NW-CHILD Study. Perceptual and Motor Skills, 125(5), 966-988. https://doi.org/ 10.1177/0031512518786806

Di Lieto, M. C., Inguaggiato, E., Castro, E., Cecchi, F., Cioni, G., Dell'Omo, M., Laschi, C., Pecini, C., Santerini, G., Sgandurra, G., \& Dario, P. (2017). Educational Robotics intervention on Executive Functions in preschool children: A pilot study. Computers in Human Behavior, 71, 16-23. https://doi.org/10.1016/j.chb.2017.01.018 
Di Rosa, G., Cavallaro, T., Alibrandi, A., Marseglia, L., Lamberti, M., Giaimo, E., Nicotera, A., Bonsignore, M., \& Gagliano, A. (2016). Predictive role of early milestones-related psychomotor profiles and long-term neurodevelopmental pitfalls in preterm infants. Early Human Development, 101, 49-55. https://doi.org/10.1016/j. earlhumdev.2016.04.012

Donnelly, J. E., Hillman, C. H., Castelli, D., Etnier, J. L., Lee, S., Tomporowski, P., Lambourne, K., \& Szabo-Reed, A. N. (2016). Physical activity, fitness, cognitive function, and academic achievement in children: A systematic review. In Medicine and Science in Sports and Exercise (Vol. 48, Issue 6, pp. 1197-1222). Lippincott Williams and Wilkins. https://doi.org/10.1249/ MSS.0000000000000901

Duarte-Torrado, O. E., Solovieva, Y. \& Quintanar-Rojas, L. (2018). Análisis comparativo de la adquisición del proceso lectoescritor ante distintos métodos de enseñanza. Neuropsicología Latinoamericana, 10(2), 11-19. https://www. neuropsicolatina.org/index.php/Neuropsicologia_Latinoamericana/article/view/401/237

Elaklouk, A. M., \& Zin, N. A. M. (2018). Design and usability evaluation of rehabilitation gaming system for cognitive deficiencies. Proceedings of the 2017 6th International Conference on Electrical Engineering and Informatics: Sustainable Society Through Digital Innovation, ICEEI 2017, 2017-Novem, 1-6. https://doi.org/10.1109/ ICEEI.2017.8312454

ElGarhy, S., \& Liu, T. (2016). Effects of psychomotor intervention program on students with autism spectrum disorder. School Psychology Quarterly, 31(4), 491-506. https://doi.org/10.1037/spq 0000164

Fernandes, M. C. C., Ciasca, S. M., Capelatto, I. V, \& Salgado-Azoni, C. A. (2019). Effect of a psychomotor intervention program for children with ADHD. Estudos de Psicologia, 24(1), 1-11. https://doi.org/10.22491/1678-4669. 20190001
Fonseca-Estupiñan, G. P., Rodríguez-Barreto, L. C. \& Parra-Pulido, J. H. (2016). Relación entre funciones ejecutivas y rendimiento académico por asignaturas en escolares de 6 a 12 años. Hacia La Promoción de La Salud, 21(2), 41-58. https:// doi.org/10.17151/hpsal.2016.21.2.4

Frank, J. L., Broderick, P. C., Oh, Y., Mitra, J., Kohler, K., Schussler, D. L., Geier, C., Roeser, R. W., Berrena, E., Mahfouz, J., Levitan, J., \& Greenberg, M. T. (2021). The Effectiveness of a Teacher-Delivered Mindfulness-Based Curriculum on Adolescent Social-Emotional and Executive Functioning. Mindfulness. https:// doi.org/10.1007/s12671-021-01594-9

Fuster, J. M. (2017). Prefrontal Executive Functions Predict and Preadapt. In Executive Functions in Health and Disease (pp. 3-19). Elsevier Inc. https://doi.org/10.1016/B978-0-12-803676-1. 00001-5

Galuschka, K., Görgen, R., Kalmar, J., Haberstroh, S., Schmalz, X., \& Schulte-Körne, G. (2020). Effectiveness of spelling interventions for learners with dyslexia: A meta-analysis and systematic review. Educational Psychologist, 55(1), 1-20. https://doi.org/10.1080/00461520.201 9.1659794

Ganapathy-Sankar, U., \& Monisha, R. (2020). Evaluation of perceptual ability in children with delayed language development (Dld)-a pilot study. International Journal of Pharmaceutical Research, 12(3), 2567-2569. https://doi.org/10.31838/ ijpr/2020.12.03.361

Glejzer, C., Ciccarelli, A., Maldonado, A., Bulit, F., Chomnalez, M., Facchinetti, C. \& Ricci, A. (2017). Las bases biológicas del aprendizaje ( $3^{\mathrm{a}}$ ed.). Universidad de Buenos Aires. Editorial de la Facultad de Filosofía y Letras.

Hedenius, M., Lum, J. A. G., \& Bölte, S. (2020). Alterations of procedural memory consolidation in children with developmental dyslexia. Neuropsychology, 35(2), 185-196. https://doi. org/10.1037/neu0000708 
Higashionna, T., Iwanaga, R., Tokunaga, A., Nakai, A., Tanaka, K., Nakane, H., \& Tanaka, G. (2017). Relationship between Motor Coordination, Cognitive Abilities, and Academic Achievement in Japanese Children with Neurodevelopmental Disorders. Hong Kong Journal of Occupational Therapy, 30(1), 49-55. https://doi.org/10.1016/j. hkjot.2017.10.002

Ibáñez-Azorín, E., Martin-Lobo, P., Vergara-Moragues, E., \& Calvo, A. (2018). Neuropsychological program of English learning for students with dyslexia. Electronic Journal of Research in Educational Psychology, 16(45), 417-445. https://doi. org/10.25115/ejrep.v16i45.2100

Ison, M. S. \& Korzeniowski, C. (2016). El rol de la atención y percepción viso-espacial en el desempeño lector en la mediana infancia. Psykhe, 25(1), 1-13. https://doi.org/10.7764/psykhe.25.1.761

Janke, K. M., Klein-Tasman, B. P., Garwood, M. M., Davies, W. H., Trapane, P., \& Holman, K. S. (2014). Relations Between Executive Functioning and Academic Performance in Adolescents with Neurofibromatosis-1. Journal of Developmental and Physical Disabilities, 26(4), 431-450. https://doi.org/10.1007/s10882-014-9375-3

Jassim, N., Baron-Cohen, S., \& Suckling, J. (2021). Meta-analytic evidence of differential prefrontal and early sensory cortex activity during non-social sensory perception in autism. Neuroscience \& Biobehavioral Reviews. https://doi.org/10.1016/j. neubiorev.2021.04.014

Keenan, L., Conroy, S., O’Sullivan, A., \& Downes, M. (2019). Executive functioning in the classroom: Primary school teachers' experiences of neuropsychological issues and reports. Teaching and Teacher Education, 86, 102912. https:// doi.org/10.1016/j.tate.2019.102912

Kuhl, U., Neef, N. E., Kraft, I., Schaadt, G., Dörr, L., Brauer, J., Czepezauer, I., Müller, B., Wilcke, A., Kirsten, H., Emmrich, F., Boltze, J., Friederici, A. D., \& Skeide, M. A. (2020). The emergence of dyslexia in the developing brain. NeuroImage,
211, 116633. https://doi.org/10.1016/j.neuroimage.2020.116633

Libertus, K., \& Hauf, P. (2017). Editorial: Motor skills and their foundational role for perceptual, social, and cognitive development. In Frontiers in Psychology (Vol. 8, Issue MAR). Frontiers Research Foundation. https://doi.org/10.3389/ fpsyg.2017.00301

Libertus, K., Wilson, P. H., \& Leonard, H. C. (2016). The Impact of Poor Motor Skills on Perceptual, Social and Cognitive Development: The Case of Developmental Coordination Disorder. Frontiers in Psychology | Www. Frontiersin.Org, 1, 311. https://doi.org/10.3389/fpsyg.2016.00311

Lozoya-Meza, E., Amaya Gutiérrez, S. \& Lozoya Ocegueda, R. (2018). La neurociencia cognitiva en la formación inicial de docentes investigadores educativos. Ciencia y Educación, 2(3), 11-25. https://doi.org/10.22206/cyed.2018. v2i3. pp11-25

Macdonald, K., Milne, N., Orr, R., \& Pope, R. (2018). Relationships Between Motor Proficiency and Academic Performance in Mathematics and Reading in School-Aged Children and Adolescents: A Systematic Review. International Journal of Environmental Research and Public Health, 15(8), 1603. https://doi.org/10.3390/ ijerph 15081603

Magalhães, S., Carneiro, L., Limpo, T., \& Filipe, M. (2020). Executive functions predict literacy and mathematics achievements: The unique contribution of cognitive flexibility in grades 2, 4, and 6. Child Neuropsychology, 26(7), 934-952. https://doi.org/10.1080/09297049. 2020.1740188

Martín-Lobo, P. (2015). Procesos y programas de neuropsicología educativa. Secretaría General Técnica. Centro de Publicaciones. Ministerio de Educación, Cultura y Deporte. https://sede. educacion.gob.es/publiventa/procesos-y-programas-de-neuropsicologia-educativa/ensenanza-neurologia/20612 
Merchán-Price, M. S., \& Henao-Calderon, J. L. (2011). Influencia de la percepción visual en el aprendizaje. Ciencia \& Tecnología Para La Salud Visual y Ocular, 9(1), 93-101. https:// doi.org/10.19052/sv.221

Moore, D. R., Ferguson, M. A., Edmondson-Jones, A. M., Ratib, S., \& Riley, A. (2010). Nature of auditory processing disorder in children. Pediatrics, 126(2). https://doi.org/10.1542/ peds.2009-2826

Murphy, P. K., \& Benton, S. L. (2010). The new frontier of educational neuropsychology: Unknown opportunities and unfulfilled hopes. Contemporary Educational Psychology, 35(2), 153-155. https://doi.org/https://doi.org/10.1016/j. cedpsych.2010.04.006

Padua-Rodríguez, L. M. (2019). Factores individuales y Familiares asociados al bajo rendimiento académico en estudiantes universitarios. Revista Mexicana de Investigacion Educativa, 24(80), 173-195. https://dialnet.unirioja.es/servlet/articulo? codigo $=6987382 \&$ info $=$ resumen $\&$ idio $\mathrm{ma}=\mathrm{SPA}$

Roberts, G. J., Cho, E., Garwood, J. D., Goble, G. H., Robertson, T., \& Hodges, A. (2020). Reading Interventions for Students with Reading and Behavioral Difficulties: a Meta-analysis and Evaluation of Co-occurring Difficulties. In Educational Psychology Review (Vol. 32, Issue 1, pp. 17-47). Springer. https://doi.org/10.1007/s10648-01909485-1

Rufo-Campos, M. (2006). La neuropsicología: Historia, conceptos básicos y aplicaciones. Revista de Neurología, 43(SUPPL. 1), S057. https://doi. org/10.33588/rn.43s01.2006392

Santner, A., Kopp, M., \& Federolf, P. (2018). Partly randomised, controlled study in children aged 6-10 years to investigate motor and cognitive effects of a 9-week coordination training intervention with concurrent mental tasks. $B M J$ Open, 8(5). https://doi.org/10.1136/bmjopen2017-021026
Senese, V. P., Zappullo, I., Baiano, C., Zoccolotti, P., Monaco, M., \& Conson, M. (2020). Identifying neuropsychological predictors of drawing skills in elementary school children. Child Neuropsychology, 26(3), 345-361. https://doi. org/10.1080/09297049.2019.1651834

Siddiqua, A., Duku, E., Georgiades, K., Mesterman, R., \& Janus, M. (2020). Neighbourhood-level prevalence of teacher-reported Autism Spectrum Disorder among kindergarten children in Canada: A population level study. SSM Population Health, 10, 100520. https:/doi. org/10.1016/j.ssmph.2019.100520

Sotomayor-León, K. N., Merizalde-Yperti, N., BorjaOchoa, J.L. \& Jurado-Vasquez, P. P. (2020). Implementación de la sala multisensorial Snoezelen, favorecedora de la percepción de sensaciones y desarrollo de procesos de enseńanza-aprendizaje en niños con fracaso escolar de 6 a 10 años. Polo Del Conocimiento, 5(10), 616-632. https://doi. org/10.23857/pc.v5i10.1836

Valencia-Echeverry, J., García-Murcia, D. C., Londoño-Martínez, J. D. \& Barrera-Valencia, M. A. (2020). Habilidades gnósico-práxicas relacionadas con dificultades del aprendizaje de la lectura y la escritura en individuos de 9 a 12 años. CES Psicología, 13(2 SE-), 113-128. https://revistas.ces.edu.co:443/index.php/ psicologia/article/view/5040

Vasquez, E., \& Marino, M. T. (2021). Enhancing Executive Function While Addressing Learner Variability in Inclusive Classrooms. Intervention in School and Clinic, 56(3), 179-185. https:// doi.org/10.1177/1053451220928978

Ventura, L. M., Grieco, J. A., Evans, C. L., Kuhlthau, K. A., MacDonald, S. M., Tarbell, N. J., Yock, T. I., \& Pulsifer, M. B. (2018). Executive functioning, academic skills, and quality of life in pediatric patients with brain tumors post-proton radiation therapy. Journal of Neuro-Oncology, 137(1), 119-126. https://doi. org/10.1007/s1 1060-017-2703-6 
Watson, A., Timperio, A., Brown, H., Best, K., \& Hesketh, K. D. (2017). Effect of classroom-based physical activity interventions on academic and physical activity outcomes: A systematic review and meta-analysis. In International Journal of Behavioral Nutrition and Physical Activity (Vol. 14, Issue 1). BioMed Central Ltd. https://doi. org/10.1186/s12966-017-0569-9
Werker, J. F. (2018). Perceptual beginnings to language acquisition. In Applied Psycholinguistics (Vol. 39, Issue 4, pp. 703-728). Cambridge University Press. https://doi.org/10.1017/ S0142716418000152 